\title{
MEROMORPHIC SOLUTIONS OF CERTAIN FUNCTIONAL EQUATIONS
}

\author{
Mingbo Yang and Ping Li
}

\begin{abstract}
By utilizing Nevanlinna's value distribution theory, we study the existence or solvability of meromorphic solutions of functional equations of the type $P(f) f^{\prime} P(g) g^{\prime}=1$, where $P(z)$ is a polynomial with two distinct zeros at least. We show that such type of equations have no meromorphic solutions $f$ and $g$ when $P(z)$ has at least three distinct zeros. Moreover, for some polynomials $P(z)$ with two distinct zeros only, such type of equations possess transcendental meromorphic solutions which can be expressed by Weierstrass $\wp$ function.
\end{abstract}

\section{INTRODUCTION}

In this paper, meromorphic functions are always defined in the complex plane $\mathbb{C}$. Let $f(z)$ be a nonconstant meromorphic function. We shall use the standard notations in Nevanlinna's value distribution theory of meromorphic functions such as $T(r, f), m(r, f), N(r, f)$ and $\bar{N}(r, f)$ (see, e.g., [4]). We use $S(r, f)$ to denote the quantity $o(T(r, f)),(r \rightarrow \infty, r \notin E)$, where the letter $E$ is a set of $r \in(0, \infty)$ with finite linear measure. A meromorphic function $a(z)(\not \equiv \infty)$ is called a small function with respect to $f(z)$ provided that $T(r, a)=S(r, f)$.

Let $f(z)$ and $g(z)$ be two nonconstant meromorphic functions, and $c$ a finite complex number. If $f(z)-c$ and $g(z)-c$ have the same zeros counting multiplicity, then we say that $f(z)$ and $g(z)$ share the value $c$ CM. Let $a, b$ be two constants. We use $\bar{N}(r, f=a, g=b)$ to denote the reduced counting function of the common zeros of $f-a$ and $g-b$. If

$$
\bar{N}\left(r, \frac{1}{f-a}\right)-\bar{N}(r, f=a, g=a)=S(r, f),
$$

Received October 2, 2008, accepted November 20, 2009.

Communicated by Der-Chen Chang.

2000 Mathematics Subject Classification: 30D35, 30D05.

Key words and phrases: Meromorphic function, Functional equation, Uniqueness.

Project 10871089 supported by NSFC. 
and

$$
\bar{N}\left(r, \frac{1}{g-a}\right)-\bar{N}(r, f=a, g=a)=S(r, g),
$$

then we say that $f$ and $g$ share the value $a \mathrm{CM}^{*}$. It is obvious that $f$ and $g$ share $a \mathrm{CM}$ implies that $f$ and $g$ share $a \mathrm{CM}^{*}$.

Nevanlinna's value distribution theory has been used to study the Fermat type of equations of meromorphic functions since 1960s (see e.g. [2, 8]). And we refer the reader to [3,5] and [10] for some recent developments of value sharing and more general type equation $P(f)=Q(g)$ of meromorphic functions, where $P, Q$ are two polynomials in $\mathbb{C}[z]$.

In 1977, C.-C. Yang and X.-H. Hua [9] proved the following theorem.

Theorem A. Suppose that $f, g$ are two nonconstant meromorphic functions and $n \geq 6$ is an integer. If $f^{n} f^{\prime} g^{n} g^{\prime}=1$, then $g(z)=c_{1} e^{c z}$ and $f(z)=c_{2} e^{-c z}$, where $c, c_{1}$ and $c_{2}$ are constants satisfying $\left(c_{1} c_{2}\right)^{n+1} c^{2}=-1$.

In this paper, by using Nevanlinna's value distribution theory, we study the existence or solvability of the meromorphic solutions of functional equations of the type

$$
P(f) f^{\prime} P(g) g^{\prime}=1,
$$

where $P$ is a polynomial with two distinct zeros at least. We shall prove the following result.

Theorem 1. Suppose that $P(z)$ is a polynomial with three distinct zeros. Then equation (1) has no meromorphic solutions $f$ and $g$.

We point out that equation (1) may or may not have meromorphic solutions, if the polynomial $P$ just has two distinct zeros. In fact, we can prove the following results.

Theorem 2. Let $d$ be a nonzero constant, $k$ be a positive integer. Then the functional equation

$$
f^{2 k+1}(f-1)^{k} f^{\prime} g^{2 k+1}(g-1)^{k} g^{\prime}=d
$$

has no nonconstant meromorphic solution.

Theorem 3. Suppose that $c$ is a nonzero constant, $k$ is a positive integer. Then the pair $(f, g)$ of nonconstant meromorphic solution of the following equation

$$
(f-1)^{k}(f+1)^{k} f^{\prime}(g-1)^{k}(g+1)^{k} g^{\prime}=c
$$


must be

$$
\begin{gathered}
f=1+\frac{(\wp-c / 2)^{2}}{c \wp}, \\
g=-\frac{\wp^{2}+c^{2} / 4}{(\wp-c / 2)(\wp+c / 2)},
\end{gathered}
$$

where $\wp$ is the $\wp$-function satisfies

$$
\left(\wp^{\prime}\right)^{2}=\wp(\wp-c / 2)(\wp+c / 2) .
$$

As an application of Theorem 1, we prove the following result.

Corollary 1. Suppose that $f$ and $g$ are two nonconstant meromorphic functions. Let $m, n$ be two relatively prime positive integers satisfying $n+m \geq 18$, and $a, b$ two distinct constants. Let $H(z)=(z-a)^{n}(z-b)^{m}$. If the derivatives of the two functions $H(f)$ and $H(g)$ share $1 C M$, then

$$
f=\frac{b h^{m+n}+(a-b) h^{m}-a}{h^{m+n}-1}, \quad g=\frac{a h^{m+n}+(b-a) h^{m}-b}{h^{m+n}-1},
$$

where $h$ is a nonconstant meromorphic function.

\section{LEMMAS}

The following lemmas will be used in the proofs of our theorems. Lemma 1 can be derived easily by the lemma of logarithmic derivative, i.e., $m\left(r, f^{\prime} / f\right)=S(r, f)$, see e.g. [4]. Lemma 2 is well-known.

Lemma 1. Let $f(z)$ be a nonconstant meromorphic function, and let $P_{k}(f)$ be a polynomial in $f$ of degree $k$, and $a_{i}, i=1,2 \ldots, n$ be distinct complex numbers in $\mathbb{C}$, and $j$ be a positive integer. Let

$$
g=\frac{P_{k}(f) f^{(j)}}{\left(f-a_{1}\right) \cdots\left(f-a_{n}\right)} .
$$

If $k<n$, then $m(r, g)=S(r, f)$.

Lemma 2. ([11]). Let $f(z)$ be a nonconstant meromorphic function. If

$$
R(f)=\frac{P_{1}(f)}{Q_{1}(f)}=\frac{a_{p} f^{p}+a_{p-1} f^{p-1}+\cdots+a_{0}}{b_{q} f^{q}+b_{q-1} f^{q-1}+\cdots+b_{0}}
$$

where $P_{1}(f)$ and $Q_{1}(f)$ are two relatively prime polynomials of degree $p$ and $q$, respectively, and all the coefficients $a_{i}(z)$ and $b_{j}(z)$ are small functions of $f(z)$ with $a_{p}(z) \not \equiv 0, b_{q}(z) \not \equiv 0$, then we have

$$
T(r, R(f))=\max \{p, q\} T(r, f)+S(r, f) .
$$


Lemma 3. ([6] or [7]). Suppose that $f$ and $g$ are two nonconstant meromorphic functions sharing the value $1 C M$. If $f \neq g$ and $f g \neq 1$, then the following inequality holds:

(8) $T(r, f) \leq N_{2}(r, f)+N_{2}(r, g)+N_{2}\left(r, \frac{1}{f}\right)+N_{2}\left(r, \frac{1}{g}\right)+S(r, f)+S(r, g)$, where $N_{2}(r, f)=\bar{N}(r, f)+\bar{N}_{(2}(r, f)$.

Lemma 4. ([1] or [2]). Any functions $F(z)$ and $G(z)$, which are meromorphic in the plane and satisfy $F^{3}+G^{3}=1$, have the form

$$
F=\frac{1}{2 \sqrt{3}} \frac{\sqrt{3}+\wp^{\prime}(h)}{\wp(h)}, G=\frac{\eta}{2 \sqrt{3}} \frac{\sqrt{3}-\wp^{\prime}(h)}{\wp(h)},
$$

where $\eta$ is a cube-root of unity, $h$ is a nonconstant entire function, and $\wp$ is the $\wp$-function satisfying

$$
\left(\wp^{\prime}\right)^{2}=4 \wp^{3}-1
$$

Lemma 5. Let $c$ be a nonzero constant. Then the meromorphic solution of the equation

$$
\left(f^{\prime}\right)^{4}=c^{2}(f-1)^{3}(f+1)^{3},
$$

must be the following function

$$
f=1+\frac{(\wp-c / 2)^{2}}{c \wp}
$$

where $\wp$ is the $\wp$-function satisfying

$$
\left(\wp^{\prime}\right)^{2}=\wp(\wp-c / 2)(\wp+c / 2) .
$$

Proof. Suppose that $f$ is a meromorphic solution of equation (10). It is easily seen that the function $c f\left(f^{2}-1\right)-\left(f^{\prime}\right)^{2}$ cannot be identically zero. Let

$$
g=\frac{\left(c^{2} / 2\right)\left(f^{2}-1\right)}{c f\left(f^{2}-1\right)-\left(f^{\prime}\right)^{2}} .
$$

Then we have

$$
\left(f^{\prime}\right)^{2}=\frac{(c / 2)\left(f^{2}-1\right)(2 f g-c)}{g} .
$$


It follows from (10) and (14) that $f^{2}-1=(f-c /(2 g))^{2}$. Therefore,

$$
f=1+\frac{(g-c / 2)^{2}}{c g}
$$

and thus

$$
f^{\prime}=\frac{g^{\prime}(g-c / 2)(g+c / 2)}{c g^{2}} .
$$

By (15), we have

$$
2 f g-c=(2 / c)(g-c / 2)(g+c / 2) .
$$

By substituting (15), (16) and (17) into (14), we get $\left(g^{\prime}\right)^{2}=g(g-c / 2)(g+c / 2)$. This also completes the proof of Lemma 5.

\section{Proof of Theorem 1}

Suppose that $(f, g)$ is a pair of nonconstant meromorphic solution of equation (1), where $P(z)$ has three distinct zeros $r_{1}, r_{2}, r_{3}$. Let $P(z)=\left(z-r_{1}\right)^{k_{1}}(z-$ $\left.r_{2}\right)^{k_{2}}\left(z-r_{3}\right)^{k_{3}} Q(z)$, where $Q(z)$ is a polynomial of degree $p$. If $z$ is a $r_{1}$ point of $f$ of multiplicity $m$, then it is a pole of $g$ of multiplicity $n$, and $m k_{1}+m-1=$ $n\left(k_{1}+k_{2}+k_{3}+p\right)+n+1$. Therefore, $m \geq\left(k_{1}+k_{2}+k_{3}+3+p\right) /\left(k_{1}+1\right)$. Similarly, the multiplicities of all $r_{i}$ points of $f$ are greater than or equal to $n_{i}:=$ $\left(k_{1}+k_{2}+k_{3}+3+p\right) /\left(k_{i}+1\right), i=1,2,3$. If $p>0$, then $1 / n_{1}+1 / n_{2}+1 / n_{3}<1$. By Nevanlinna's second fundamental theorem, we have

$$
\begin{aligned}
T(r, f) & \leq \bar{N}\left(r, \frac{1}{f-r_{1}}\right)+\bar{N}\left(r, \frac{1}{f-r_{2}}\right)+\bar{N}\left(r, \frac{1}{f-r_{3}}\right)+S(r, f) \\
& \leq \frac{1}{n_{1}} N\left(r, \frac{1}{f-r_{1}}\right)+\frac{1}{n_{2}} N\left(r, \frac{1}{f-r_{2}}\right)+\frac{1}{n_{3}} N\left(r, \frac{1}{f-r_{3}}\right)+S(r, f) \\
& \leq\left(\frac{1}{n_{1}}+\frac{1}{n_{2}}+\frac{1}{n_{3}}\right) T(r, f)+S(r, f),
\end{aligned}
$$

Therefore, $1 / n_{1}+1 / n_{2}+1 / n_{3} \geq 1$, a contradiction. Hence $p=0$, i.e., $Q(z)$ is a nonzero constant. The last inequality above also shows that $f$ has no Nevanlinna exceptional value. Let $m_{i}:=\left(k_{1}+k_{2}+k_{3}+3\right) /\left(k_{i}+1\right), i=1,2,3$. Then the multiplicities of all $r_{i}$ points of $f$ are greater than or equal to $m_{i}$. If $m_{1}$ is not an integer, then the multiplicities of all $r_{1}$ points of $f$ are greater than or equal to $\left[m_{1}\right]+1$. Since $1 /\left(\left[m_{1}\right]+1\right)+1 / m_{2}+1 / m_{3}<1$, we can still derive a contradiction by Nevanlinna's second fundamental theorem. Hence $m_{1}$ is a positive integer. Similarly, $m_{2}$ and $m_{3}$ are positive integers, too. 
Without loss of generality, we assume $m_{1} \leq m_{2} \leq m_{3}$. Since

$$
k_{1}+k_{2}+k_{3}+3=m_{i}\left(k_{i}+1\right), i=1,2,3,
$$

we have $3\left(k_{1}+k_{2}+k_{3}+3\right) \geq m_{1}\left(k_{1}+k_{2}+k_{3}+3\right)$, that is $m_{1} \leq 3$. Note that

$$
\frac{1}{m_{1}}+\frac{1}{m_{2}}+\frac{1}{m_{3}}=1 \text {. }
$$

We have $m_{1}>1$. Therefore, $m_{1}=2$ or $m_{1}=3$.

If $m_{1}=3$, then by $m_{1} \leq m_{2} \leq m_{3}$ and (19) we get $m_{1}=m_{2}=m_{3}=3$. Therefore, there exists a positive integer $k$ such that $k_{i}=k, i=1,2,3$.

If $m_{1}=2$, then we have $k_{1}=k_{2}+k_{3}+1$, by (18) and $m_{1} \leq m_{2} \leq m_{3}$, we get $2\left(k_{1}+k_{2}+k_{3}+3\right) \geq m_{2}\left(k_{2}+k_{3}+2\right)$. Therefore, $4\left(k_{2}+k_{3}+2\right) \geq m_{2}\left(k_{2}+k_{3}+2\right)$, and thus $m_{2} \leq 4$. From (19), it is easily seen that $m_{2} \neq 2$. Hence we have $m_{2}=3$ or $m_{2}=4$. If $m_{2}=3$, then we have $m_{3}=6$. Thus there exists a positive integer $k$ such that $k_{1}=3 k+2, k_{2}=2 k+1, k_{3}=k$. If $m_{2}=4$, then we have $m_{3}=4$, Thus there exists a positive integer $k$ such that $k_{1}=2 k+1, k_{2}=k, k_{3}=k$. Hence equation (1) becomes one of the following three equations:

$$
\begin{gathered}
\left(f-r_{1}\right)^{k}\left(f-r_{2}\right)^{k}\left(f-r_{3}\right)^{k} f^{\prime}\left(g-r_{1}\right)^{k}\left(g-r_{2}\right)^{k}\left(g-r_{3}\right)^{k} g^{\prime}=d ; \\
\left(f-r_{1}\right)^{2 k+1}\left(f-r_{2}\right)^{k}\left(f-r_{3}\right)^{k} f^{\prime}\left(g-r_{1}\right)^{2 k+1}\left(g-r_{2}\right)^{k}\left(g-r_{3}\right)^{k} g^{\prime}=d ; \\
\left(f-r_{1}\right)^{3 k+2}\left(f-r_{2}\right)^{2 k+1}\left(f-r_{3}\right)^{k} f^{\prime}\left(g-r_{1}\right)^{3 k+2}\left(g-r_{2}\right)^{2 k+1}\left(g-r_{3}\right)^{k} g^{\prime}=d,
\end{gathered}
$$

Since all $r_{j}$ points $(j=1,2,3)$ of $f$ are poles of $g$. By Nevanlinna's second fundamental theorem, we have $T(r, f) \leq T(r, g)+S(r, f)$. Symmetrically, we have $T(r, g) \leq T(r, f)+S(r, g)$. Hence $T(r, f)=T(r, g)+S(r)$, where $S(r):=$ $S(r, f)=S(r, g)$. By the above discussion, we see that the multiplicities of all $r_{i}$ points of $f$ are greater than or equal to $m_{i}$. By Nevanlinna's second fundamental theorem, we have

$$
\begin{aligned}
T(r, f) & \leq \bar{N}\left(r, \frac{1}{f-r_{1}}\right)+\bar{N}\left(r, \frac{1}{f-r_{2}}\right)+\bar{N}\left(r, \frac{1}{f-r_{3}}\right)+S(r) \\
& \leq \frac{1}{m_{1}} N\left(r, \frac{1}{f-r_{1}}\right)+\frac{1}{m_{2}} N\left(r, \frac{1}{f-r_{2}}\right)+\frac{1}{m_{3}} N\left(r, \frac{1}{f-r_{3}}\right)+S(r) \\
& \leq T(r, f)+S(r),
\end{aligned}
$$

which implies that

$$
N\left(r, \frac{1}{f-r_{i}}\right)=m_{i} \bar{N}\left(r, \frac{1}{f-r_{i}}\right)+S(r) \neq S(r), i=1,2,3 .
$$


Therefore, "almost all" $r_{i}$ points of $f$ have multiplicity $m_{i}$, and thus "almost all" poles of $g$ are simple. Symmetrically, "almost all" $r_{i}$ points of $g$ have multiplicity $m_{i}$, and "almost all" poles of $f$ are simple. The above equation also shows that $r_{i}$ and $\infty$ cannot be the exceptional values of $f$ and $g$.

In the following, we study the three functional equations (20), (21) and (22), respectively.

\subsection{Solution of equation (20)}

By a transformation, we need to study equation

$$
f^{k}\left(f-r_{1}\right)^{k}\left(f-r_{2}\right)^{k} f^{\prime} g^{k}\left(g-r_{1}\right)^{k}\left(g-r_{2}\right)^{k} g^{\prime}=d
$$

only, where $d, r_{1}, r_{2}$ are nonzero constant, and $r_{1} \neq r_{2}$. Suppose that $f$ and $g$ are nonconstant meromorphic functions of the equation. Then the multiplicities of "almost all" zeros, $r_{1}$ points and $r_{2}$ points of $f$ and $g$ are 3, and "almost all" the poles of $f$ and $g$ are simple. Let

$$
\varphi_{1}=\frac{\left(f^{\prime}\right)^{3}}{f^{2}\left(f-r_{1}\right)^{2}\left(f-r_{2}\right)^{2}}, \quad \varphi_{2}=\frac{\left(g^{\prime}\right)^{3}}{g^{2}\left(g-r_{1}\right)^{2}\left(g-r_{2}\right)^{2}} .
$$

Then we have $\varphi_{i} \not \equiv 0$ and $N\left(r, \varphi_{i}\right)=S(r), i=1,2$. We can rewrite the first equation in (24) as

$$
\varphi_{1}=\frac{f^{\prime}}{f\left(f-r_{1}\right)} \cdot \frac{f^{\prime}}{f\left(f-r_{2}\right)} \cdot \frac{f^{\prime}}{\left(f-r_{1}\right)\left(f-r_{2}\right)} .
$$

By Lemma 1, we get $m\left(r, \varphi_{1}\right)=S(r)$. Therefore, $T\left(r, \varphi_{1}\right)=S(r)$. Similarly, we have $T\left(r, \varphi_{2}\right)=S(r)$. By rewriting the first equation in (24) as

$$
f=\frac{1}{\varphi_{1}} \frac{f^{\prime}}{f}\left(\frac{f^{\prime}}{\left(f-r_{1}\right)\left(f-r_{2}\right)}\right)^{2}
$$

and by Lemma 1, we get $m(r, f)=S(r)$. Similarly, we have $m(r, g)=S(r)$. Let

$$
\varphi=f\left(f-r_{1}\right)\left(f-r_{2}\right) g\left(g-r_{1}\right)\left(g-r_{2}\right) .
$$

It is obvious that $\varphi \not \equiv 0$. From (23), we see that poles of $f$ must be zeros of $g^{2}\left(g-r_{1}\right)^{2}\left(g-r_{2}\right)^{2} g^{\prime}$. The second equation in (24) tell us that zeros of $g^{\prime}$ must be zeros of $\varphi_{2}$ provided that they are not the zeros of $g^{2}\left(g-r_{1}\right)^{2}\left(g-r_{2}\right)^{2}$. Hence "almost all" poles of $f$ are zeros of $g^{2}\left(g-r_{1}\right)^{2}\left(g-r_{2}\right)^{2}$. Symmetrically, "almost all" poles of $g$ are zeros of $f^{2}\left(f-r_{1}\right)^{2}\left(f-r_{2}\right)^{2}$. This investigation shows that $N(r, \varphi)=S(r)$. Hence $T(r, \varphi)=S(r)$. By equation (23), we get

$$
\varphi^{k} f^{\prime} g^{\prime}=d \text {. }
$$


Suppose that $z_{1}$ is a zero of $g$ of multiplicity 3 . Then $z_{1}$ must be a simple pole of $f$. We have the following Laurent expansions in a neighborhood of $z_{1}$,

$$
f(z)=\frac{A_{1}}{z-z_{1}}+O(1), \quad g(z)=A_{2}\left(z-z_{1}\right)^{3}+O\left(\left(z-z_{1}\right)^{4}\right),
$$

where $A_{1}$ and $A_{2}$ are nonzero constants. By taking derivatives in the above expansions, we get

$$
f^{\prime}(z)=\frac{-A_{1}}{\left(z-z_{1}\right)^{2}}+O(1), \quad g^{\prime}(z)=3 A_{2}\left(z-z_{1}\right)^{2}+O\left(\left(z-z_{1}\right)^{3}\right) .
$$

From (26), we get

$$
\varphi^{k}\left(z_{1}\right)\left(-3 A_{1} A_{2}\right)=d .
$$

From (25), we get

$$
\varphi\left(z_{1}\right)=A_{1}^{3} A_{2} r_{1} r_{2} .
$$

On the other hand, from the first equation in (24), we get

$$
\varphi\left(z_{1}\right) \varphi_{1}\left(z_{1}\right)=-A_{2} r_{1} r_{2}
$$

Therefore,

$$
A_{1}^{3}=-\frac{1}{\varphi_{1}\left(z_{1}\right)}, \quad A_{2}=-\frac{\varphi\left(z_{1}\right) \varphi_{1}\left(z_{1}\right)}{r_{1} r_{2}} .
$$

From (27) and the above two equations, we obtain

$$
\varphi^{3 k}\left(z_{1}\right)\left(-\frac{27}{\varphi_{1}\left(z_{1}\right)} \frac{\varphi^{3}\left(z_{1}\right) \varphi_{1}^{3}\left(z_{1}\right)}{r_{1}^{3} r_{2}^{3}}\right)=d^{3} .
$$

It follows that

$$
27 \varphi^{3 k+3}\left(z_{1}\right) \varphi_{1}^{2}\left(z_{1}\right)=-\left(d r_{1} r_{2}\right)^{3},
$$

which means that $z_{1}$ is a zero of the function $27 \varphi^{3 k+3} \varphi_{1}^{2}+\left(d r_{1} r_{2}\right)^{3}$. Note that this function is small with respect to $f$ and $g$. If $27 \varphi^{3 k+3} \varphi_{1}^{2} \not \equiv-\left(d r_{1} r_{2}\right)^{3}$, then we get $N(r, 1 / g)=S(r)$. This means that 0 is an Nevanlinna exceptional value of $g$, which is impossible. Hence

$$
27 \varphi^{3 k+3} \varphi_{1}^{2}=-\left(d r_{1} r_{2}\right)^{3} .
$$

Similarly we have

$$
27 \varphi^{3 k+3} \varphi_{2}^{2}=-\left(d r_{1} r_{2}\right)^{3}
$$


Therefore, $\varphi_{1}=\varphi_{2}$ or $\varphi_{1}=-\varphi_{2}$.

By (23), (24), (25) and (26), we deduce that

$$
\varphi^{3 k+2} \varphi_{1} \varphi_{2}=d^{3} \text {. }
$$

From (32) and (34), we obtain

$$
\varphi=-\frac{\varphi_{2}}{\varphi_{1}}\left(\frac{r_{1} r_{2}}{3}\right)^{3} .
$$

Note that $\varphi_{1}=\varphi_{2}$ or $\varphi_{1}=-\varphi_{2}$. The above equation implies that $\varphi$ is a nonzero constant, and thus by (32) and (33) we see that both $\varphi_{1}$ and $\varphi_{2}$ are nonzero constants.

Taking the derivative in equation (25) yields

$$
\frac{f^{\prime} L(f)}{f\left(f-r_{1}\right)\left(f-r_{2}\right)}+\frac{g^{\prime} L(g)}{g\left(g-r_{1}\right)\left(g-r_{2}\right)}=0,
$$

where $L(z)$ is a polynomial defined by

$$
L(z)=3\left(z^{2}-\frac{2}{3}\left(r_{1}+r_{2}\right) z+\frac{r_{1} r_{2}}{3}\right) .
$$

Note that zeros of $f^{\prime}$ are poles of $g$, and zeros of $g^{\prime}$ are poles of $f$. By (36), we see that $L(f)$ and $L(g)$ share $0 \mathrm{CM}$.

We distinguish two cases below:

Case (a). $r_{1}^{2}-r_{1} r_{2}+r_{2}^{2} \neq 0$.

In this case, the equation $L(z)=0$ has two distinct roots denoted by $a_{1}$ and $a_{2}$. Therefore, $L(z)=3\left(z-a_{1}\right)\left(z-a_{2}\right)$. Eq. (36) implies that $f$ and $g$ share the set $S=\left\{a_{1}, a_{2}\right\}$ CM, i.e., $f^{-1}(S)=g^{-1}(S)$ counting multiplicities. Note that

$$
\begin{aligned}
a_{1}\left(a_{1}-r_{1}\right)\left(a_{1}-r_{2}\right) & =a_{1}\left(a_{1}^{2}-\left(r_{1}+r_{2}\right) a_{1}+r_{1} r_{2}\right) \\
& =a_{1}\left(\frac{2}{3}\left(r_{1}+r_{2}\right) a_{1}-\frac{r_{1} r_{2}}{3}-\left(r_{1}+r_{2}\right) a_{1}+r_{1} r_{2}\right) \\
& =\frac{2}{3} r_{1} r_{2} a_{1}-\frac{1}{3}\left(r_{1}+r_{2}\right) a_{1}^{2} \\
& =\frac{2}{3} r_{1} r_{2} a_{1}-\frac{1}{3}\left(r_{1}+r_{2}\right)\left(\frac{2}{3}\left(r_{1}+r_{2}\right) a_{1}-\frac{r_{1} r_{2}}{3}\right) .
\end{aligned}
$$

We obtain

$$
a_{1}\left(a_{1}-r_{1}\right)\left(a_{1}-r_{2}\right)=-\frac{2}{9}\left(r_{1}^{2}-r_{1} r_{2}+r_{2}^{2}\right) a_{1}+\frac{r_{1} r_{2}\left(r_{1}+r_{2}\right)}{9}
$$

Similarly we can get

$$
a_{2}\left(a_{2}-r_{1}\right)\left(a_{2}-r_{2}\right)=-\frac{2}{9}\left(r_{1}^{2}-r_{1} r_{2}+r_{2}^{2}\right) a_{2}+\frac{r_{1} r_{2}\left(r_{1}+r_{2}\right)}{9}
$$


If both $\bar{N}\left(r, f=a_{1}, g=a_{1}\right) \neq S(r)$ and $\bar{N}\left(r, f=a_{1}, g=a_{2}\right) \neq S(r)$ hold, then by (25), we have $\varphi=\left(a_{1}\left(a_{1}-r_{1}\right)\left(a_{1}-r_{2}\right)\right)^{2}$ and $\varphi=a_{1}\left(a_{1}-\right.$ $\left.r_{1}\right)\left(a_{1}-r_{2}\right) a_{2}\left(a_{2}-r_{1}\right)\left(a_{2}-r_{2}\right)$. It follows that $a_{1}\left(a_{1}-r_{1}\right)\left(a_{1}-r_{2}\right)=a_{2}\left(a_{2}-\right.$ $\left.r_{1}\right)\left(a_{2}-r_{2}\right)$. From (38) and (39), we get $a_{1}=a_{2}$, a contradiction. Similarly, $\bar{N}\left(r, f=a_{1}, g=a_{1}\right) \neq S(r)$ and $\bar{N}\left(r, f=a_{2}, g=a_{1}\right) \neq S(r)$ cannot hold simultaneously. Therefore, the condition $\bar{N}\left(r, f=a_{1}, g=a_{1}\right) \neq S(r)$ implies $\bar{N}\left(r, f=a_{1}, g=a_{2}\right)=S(r)$ and $\bar{N}\left(r, f=a_{2}, g=a_{1}\right)=S(r)$. Accordingly, $f$ and $g$ share the value $a_{1}$ and $a_{2} \mathrm{CM}^{*}$. Hence the following function

$$
\alpha=\frac{f-a_{1}}{g-a_{1}} \frac{g-a_{2}}{f-a_{2}}
$$

satisfy $N(r, \alpha)=S(r)$ and $\alpha \not \equiv 0,1$. From (24) and by Lemma 1, we have $m\left(r, 1 / f-a_{2}\right)=S(r)$ and $m\left(r, 1 / g-a_{2}\right)=S(r)$. Therefore $m(r, \alpha)=S(r)$. Hence $T(r, \alpha)=S(r)$. It follows from the above equation that

$$
g=a_{1}+\frac{\left(a_{1}-a_{2}\right)\left(f-a_{1}\right)}{(\alpha-1) f+a_{1}-\alpha a_{2}}=\frac{\left(\alpha a_{1}-a_{2}\right) f+(1-\alpha) a_{1} a_{2}}{(\alpha-1) f+a_{1}-\alpha a_{2}} .
$$

And thus

$$
g-r_{i}=\frac{\left(\alpha a_{1}-a_{2}-(\alpha-1) r_{i}\right) f+(1-\alpha) a_{1} a_{2}-r_{i}\left(a_{1}-\alpha a_{2}\right)}{(\alpha-1) f+a_{1}-\alpha a_{2}}, i=1,2 .
$$

By substituting the above equation into (25), we get

$$
\begin{aligned}
\varphi= & f\left(f-r_{1}\right)\left(f-r_{2}\right) \frac{\left(\alpha a_{1}-a_{2}\right) f+(1-\alpha) a_{1} a_{2}}{(\alpha-1) f+a_{1}-\alpha a_{2}} \\
& \cdot \frac{\left(\alpha a_{1}-a_{2}-(\alpha-1) r_{1}\right) f+(1-\alpha) a_{1} a_{2}-r_{1}\left(a_{1}-\alpha a_{2}\right)}{(\alpha-1) f+a_{1}-\alpha a_{2}} \\
& \cdot \frac{\left(\alpha a_{1}-a_{2}-(\alpha-1) r_{2}\right) f+(1-\alpha) a_{1} a_{2}-r_{2}\left(a_{1}-\alpha a_{2}\right)}{(\alpha-1) f+a_{1}-\alpha a_{2}} .
\end{aligned}
$$

By Lemma 2 and the above equation, we deduce that $\varphi$ is not constant, a contradiction.

Similarly, if $\bar{N}\left(r, f=a_{2}, g=a_{2}\right) \neq S(r)$, then we can deduce that $f-a_{1}$ and $g-a_{2}$ share the value 0 in the sense $\mathrm{CM}^{*}$, and $f-a_{2}$ and $g-a_{1}$ share $0 \mathrm{CM}^{*}$. And thus we can show that the following function

$$
\beta=\frac{f-a_{1}}{g-a_{2}} \frac{g-a_{1}}{f-a_{2}}
$$

satisfy $T(r, \beta)=S(r)$ and $\beta \not \equiv 0$. By a similar argument as above, we can also deduce a contradiction. Hence Eq. (23) has no nonconstant meromorphic solution in Case (a). 
Case (b). $r_{1}^{2}-r_{1} r_{2}+r_{2}^{2}=0$.

In this case, the equation $L(z)=0$ has a multiple root $r=\left(r_{1}+r_{2}\right) / 3$, and $L(z)=3(z-r)^{2}$. By (36), we see that $f$ and $g$ share the value $r$ CM. Note that $r$ cannot be the exceptional value of $f$ and $g$. By (25), we get

$$
\varphi=\left(r\left(r-r_{1}\right)\left(r-r_{2}\right)\right)^{2}=\left(\frac{r_{1} r_{2}}{3}\right)^{3}=r^{6} .
$$

Combining with (35), we obtain $\varphi_{2}=-\varphi_{1}$. It follows from (32) that

$$
\varphi_{1}^{2}=-\frac{d^{3}}{r^{6(3 k+2)}} .
$$

By (24) and (36), we get

$$
\frac{f^{\prime}}{\varphi_{1}(L(f))^{2}}=\frac{g^{\prime}}{\varphi_{2}(L(g))^{2}}
$$

Since $L(z)=3(z-r)^{2}$ and $\varphi_{1}=-\varphi_{2}$, the above equation becomes

$$
\frac{f^{\prime}}{(f-r)^{4}}=-\frac{g^{\prime}}{(g-r)^{4}} \text {. }
$$

This and (26) yield

$$
\frac{\left(f^{\prime}\right)^{2}}{(f-r)^{4}}=-\frac{f^{\prime} g^{\prime}}{(g-r)^{4}}=-\frac{d}{\varphi^{k}(g-r)^{4}} .
$$

And thus

$$
\frac{f^{\prime}}{(f-r)^{2}}=\frac{A}{(g-r)^{2}},
$$

where $A$ is a constant satisfying $A^{2}=-d / \varphi^{k}$. By the above equation and the first equation in (24), we get

$$
\frac{A^{3}}{(g-r)^{6}}=\frac{\left(f^{\prime}\right)^{3}}{(f-r)^{6}}=\frac{\varphi_{1} f^{2}\left(f-r_{1}\right)^{2}\left(f-r_{2}\right)^{2}}{(f-r)^{6}} .
$$

It follows that

$$
\frac{1}{(g-r)^{3}}=M \frac{f\left(f-r_{1}\right)\left(f-r_{2}\right)}{(f-r)^{3}}
$$

where $M$ is a constant satisfying $M^{2}=\varphi_{1} / A^{3}$. On the other hand, by $\varphi=r^{6}$ and $z\left(z-r_{1}\right)\left(z-r_{2}\right)=(z-r)^{3}+r^{3}$, Eq. (25) can be rewritten as

$$
\frac{1}{(g-r)^{3}}=-\frac{1}{r^{3}} \frac{f\left(f-r_{1}\right)\left(f-r_{2}\right)}{(f-r)^{3}} .
$$


Therefore, $M=-1 / r^{3}$. And thus $A^{3}=\varphi_{1} r^{6}$. Combining with $M^{2}=\varphi_{1} / A^{3}$, we get $A=-r^{6} \varphi_{1} \varphi^{k} / d$. By (42) and (43), we get

$$
g=r+B \frac{f^{\prime}(f-r)}{f\left(f-r_{1}\right)\left(f-r_{2}\right)},
$$

where $B=\frac{d}{r^{3} \varphi_{1} \varphi^{k}}=\frac{d}{r^{6 k+3} \varphi_{1}}$.

So, if $f$ is a solution of the equation

$$
\left(f^{\prime}\right)^{3}=\varphi_{1} f^{2}\left(f-r_{1}\right)^{2}\left(f-r_{2}\right)^{2}
$$

and $g$ is the function in (44), then $(f, g)$ is a pair of solution of (23). Now we prove that Eq. (45) has no meromorphic solution. Since $r_{1}$ and $r_{2}$ satisfy $r_{1}^{2}-r_{1} r_{2}+r_{2}^{2}=0$ in the present case, we can rewrite (45) as

$$
\left(\frac{f^{\prime}}{c r}\right)^{3}+\left(\frac{r-f}{r}\right)^{3}=1
$$

where $r=\left(r_{1}+r_{2}\right) / 3$, and $c$ is a constant satisfying $c^{3}=\varphi_{1}$. By Lemma 4 , there exists a cube-root $\eta$ of unity, and a nonconstant entire function $h$ such that

$$
\frac{f^{\prime}}{c r}=\frac{1}{2 \sqrt{3}} \frac{\sqrt{3}+\wp^{\prime}(h)}{\wp(h)}, \quad \frac{r-f}{r}=\frac{\eta}{2 \sqrt{3}} \frac{\sqrt{3}-\wp^{\prime}(h)}{\wp(h)},
$$

where $\wp$ is the function satisfying (9). Taking derivative in both side of the second equation in (46), we get

$$
\frac{f^{\prime}}{r}=\frac{-\eta h^{\prime}}{2 \sqrt{3}} \cdot \frac{\wp^{\prime \prime}(h) \wp(h)+\sqrt{3} \wp^{\prime}(h)-\left(\wp^{\prime}(h)\right)^{2}}{\wp^{2}(h)} .
$$

By (9), we have $\wp^{\prime \prime}=6 \wp^{2}$. It follows that

$$
\begin{aligned}
\frac{f^{\prime}}{r} & =\frac{-\eta h^{\prime}}{2 \sqrt{3}} \frac{6 \wp^{3}(h)+\sqrt{3} \wp^{\prime}(h)-\left(\wp^{\prime}(h)\right)^{2}}{\wp^{2}(h)} \\
& =\frac{-\eta h^{\prime}}{2 \sqrt{3}} \frac{\frac{3}{2}\left[\left(\wp^{\prime}(h)\right)^{2}+1\right]+\sqrt{3} \wp^{\prime}(h)-\left(\wp^{\prime}(h)\right)^{2}}{\wp^{2}(h)} \\
& =\frac{-\eta h^{\prime}}{4 \sqrt{3}}\left(\frac{\wp^{\prime}(h)+\sqrt{3}}{\wp(h)}\right)^{2} .
\end{aligned}
$$

Combining with the first equation in (46), we get $f^{\prime}=\frac{c^{2} r \sqrt{3}}{3 \eta h^{\prime}}$, which implies that $f^{\prime}$ has no zero. And thus by (45), we see that $0, r_{1}$ and $r_{2}$ are exceptional values of $f$. This is impossible. Hence Eq. (23) has no meromorphic solution. 


\subsection{Solution of equation (21)}

The arguments in this section and the next section are very similar to that in Section 3.1, we just state the main steps and omit some of the details. In the present case, by a transformation, we need to study the equation

$$
f^{2 k+1}\left(f-r_{1}\right)^{k}\left(f-r_{2}\right)^{k} f^{\prime}\left(g^{2 k+1}\left(g-r_{1}\right)^{k}\left(g-r_{2}\right)^{k} g^{\prime}=d\right.
$$

only, where $d, r_{1}, r_{2}$ are nonzero constant and $r_{1} \neq r_{2}$. Suppose that $f$ and $g$ are nonconstant meromorphic functions satisfying Eq. (48). Then the multiplicities of "almost all" zeros of $f, g$ are 2, the multiplicities of "almost all" $r_{1}$ points and $r_{2}$ points of $f, g$ are 4, and "almost all" poles of $f, g$ are simple. Let

$$
\phi_{1}=\frac{\left(f^{\prime}\right)^{4}}{f^{2}\left(f-r_{1}\right)^{3}\left(f-r_{2}\right)^{3}}, \quad \phi_{2}=\frac{\left(g^{\prime}\right)^{4}}{g^{2}\left(g-r_{1}\right)^{3}\left(g-r_{2}\right)^{3}} .
$$

Obviously, we have $T\left(r, \phi_{i}\right)=S(r)$ and $\phi_{i} \not \equiv 0, i=1,2$. The first equation in (49) can be rewritten as

$$
f=\frac{1}{\phi_{1}} \frac{f^{\prime}}{f}\left(\frac{f^{\prime}}{\left(f-r_{1}\right)\left(f-r_{2}\right)}\right)^{3} .
$$

By Lemma 1, we have $m(r, f)=S(r)$. Similarly, we have $m(r, g)=S(r)$. Let

$$
\phi=f^{2}\left(f-r_{1}\right)\left(f-r_{2}\right) g^{2}\left(g-r_{1}\right)\left(g-r_{2}\right) .
$$

Like the arguments in Section 4.1, we can get $T(r, \phi)=S(r)$ and $\phi \not \equiv 0$. Combining with (48), we get

$$
\phi^{k} f f^{\prime} g g^{\prime}=d .
$$

By considering the Laurent expansions in the neighborhood of a zero with multiplicity 2 of $f$ and $g$, respectively, we can obtain

$$
\phi_{1}=\phi_{2}=\frac{4 d^{2}}{\left(r_{1} r_{2}\right)^{2} \phi^{2 k+1}} .
$$

On the other hand, by considering the Laurent expansions in the neighborhood of a $r_{1}$ point with multiplicity 4 of $f$, we can get

$$
\phi^{2 k+1} \phi_{1}=\frac{16 d^{2}}{\left(r_{1}-r_{2}\right)^{2} r_{1}^{2}} .
$$

From (52) and (53), we get $r_{1}=3 r_{2}$ or $r_{1}=-r_{2}$. By the symmetry of $r_{1}$ and $r_{2}$, we get $r_{1}=-r_{2}$. Let $r:=r_{1}=-r_{2}$. From (49), (50) and (51), we get

$$
\phi^{4 k+3} \phi_{1} \phi_{2}=d^{4} \text {. }
$$


From (52) and (54), we get $\phi=\frac{r^{8}}{16}$ which is a nonzero constant, and thus $\phi_{1}$ is a nonzero constant, too.

Taking $\phi=\frac{r^{8}}{16}$ and $r_{1}=-r_{2}=r$ into (50), we get

$$
f^{2}\left(f^{2}-r^{2}\right) g^{2}\left(g^{2}-r^{2}\right)=\frac{r^{8}}{16}
$$

Let $h=f g$. Then by (55), we get

$$
f^{2}+g^{2}=\frac{h^{4}-r^{4} h^{2}-\frac{r^{8}}{16}}{r^{2} h^{2}}
$$

Hence

$$
\begin{aligned}
& (f+g)^{2}=\frac{h^{4}+2 r^{2} h^{3}-r^{4} h^{2}-\frac{r^{8}}{16}}{r^{2} h^{2}}, \\
& (f-g)^{2}=\frac{h^{4}-2 r^{2} h^{3}-r^{4} h^{2}-\frac{r^{8}}{16}}{r^{2} h^{2}} .
\end{aligned}
$$

Since $r$ is a nonzero number, equation $z^{4}+2 r^{2} z^{3}-r^{4} z^{2}-\frac{r^{8}}{16}=0$ and $z^{4}-2 r^{2} z^{3}-$ $r^{4} z^{2}-\frac{r^{8}}{16}=0$ have no multiple roots, and all of the roots of the two equations are pairwise distinct. Thus by (56) and (57), we deduce that $h$ has eight multiple values. By Nevanlinna's second fundamental theorem, we know that a nonconstant meromorphic function has four multiple values at most. Therefore, $h$ must be a constant. Thus both $f+g$ and $f-g$ are constants, which implies that $f$ and $g$ are constants, a contradiction. Hence equation (48) has no nonconstant meromorphic solution.

\subsection{Solution of equation (22)}

In this case, we need to consider the equation

$$
f^{3 k+2}\left(f-r_{1}\right)^{2 k+1}\left(f-r_{2}\right)^{k} f^{\prime} g^{3 k+2}\left(g-r_{1}\right)^{2 k+1}\left(g-r_{2}\right)^{k} g^{\prime}=d
$$

only, where $d, r_{1}, r_{2}$ are nonzero constant and $r_{1} \neq r_{2}$. Suppose that $f$ and $g$ are nonconstant meromorphic functions satisfying this equation. Then the multiplicities of "almost all" zeros of $f$ and $g$ are 2, the multiplicities of "almost all" $r_{1}$ points of $f$ and $g$ are 3, the multiplicities of "almost all" $r_{2}$ points of $f$ and $g$ are 6, "almost all" poles of $f$ and $g$ are simple. Let

$$
\psi_{1}=\frac{\left(f^{\prime}\right)^{6}}{f^{3}\left(f-r_{1}\right)^{4}\left(f-r_{2}\right)^{5}}, \quad \psi_{2}=\frac{\left(g^{\prime}\right)^{6}}{g^{3}\left(g-r_{1}\right)^{4}\left(g-r_{2}\right)^{5}} .
$$

It is easily seen that $T\left(r, \psi_{i}\right)=S(r)$ and $\psi_{i} \not \equiv 0, i=1,2$. By Lemma 1, we have $m(r, f)=S(r)$ and $m(r, g)=S(r)$. Let 


$$
\psi=f^{3}\left(f-r_{1}\right)^{2}\left(f-r_{2}\right) g^{3}\left(g-r_{1}\right)^{2}\left(g-r_{2}\right),
$$

Similar to Section 4.1, we have $T(r, \psi)=S(r)$ and $\psi \not \equiv 0$. Taking (60) into (58), we get

$$
\psi^{k} f^{2}\left(f-r_{1}\right) f^{\prime} g^{2}\left(g-r_{1}\right) g^{\prime}=d .
$$

Suppose that $z_{4}$ is a zero of $f$ of multiplicity 2. Then it is a simple pole of $g$. By considering the Laurent expansions of $f$ and $g$ in a neighborhood of $z_{4}$, We can obtain

$$
\psi=\left(\frac{r_{1} r_{2}}{2}\right)^{6},
$$

and

$$
\psi_{1}=\psi_{2}=\frac{\left(d r_{1} r_{2}\right)^{3}}{\left(-2 \psi^{k+1}\right)^{3}} .
$$

Therefore, $\psi, \psi_{1}$ and $\psi_{2}$ are nonzero constant.

Suppose that $z_{5}$ is a $r_{1}$ point of $f$ of multiplicity 3 . Then it is a simple pole of $g$. By considering the Laurent expansions of $f$ and $g$ in a neighborhood of $z_{5}$, we can get

$$
\psi=\left(\frac{r_{1}\left(r_{1}-r_{2}\right)}{3}\right)^{6}
$$

and

$$
-\psi^{3 k+1}=d^{3}\left(\frac{r_{1}\left(r_{1}-r_{2}\right)}{3}\right)^{3} \frac{1}{\psi_{2}} .
$$

Combined with (63), we obtain

$$
\left(\frac{r_{2}}{2}\right)^{3}=\left(\frac{r_{1}-r_{2}}{3}\right)^{3} .
$$

Suppose that $z_{6}$ is a $r_{2}$ point of $f$ of multiplicity 6 . We see that $z_{6}$ is a simple pole of $g$. By considering the Laurent expansions of $f$ and $g$ in a neighborhood of $z_{6}$, we can deduce that

$$
\psi=\left(\frac{r_{2}\left(r_{2}-r_{1}\right)}{6}\right)^{6}
$$

and

$$
-\psi^{3 k+1}=d^{3}\left(\frac{r_{2}\left(r_{2}-r_{1}\right)}{6}\right)^{3} \frac{1}{\psi_{2}} .
$$


Combined with (63), we get

$$
\left(\frac{r_{1}}{2}\right)^{3}=\left(\frac{r_{2}-r_{1}}{6}\right)^{3}
$$

From (66) and (69), we get $r_{2}^{3}+8 r_{1}^{3}=0$. Therefore, we have $r_{2}=-2 r_{1}$ or $r_{2}^{2}=2 r_{1} r_{2}-4 r_{1}^{2}$. In both cases, we get $r_{1}=0$ from (66), which contradicts the assumption. Hence Eq. (58) has no nonconstant solution. This also completes the proof of Theorem 1.

\section{Proof of Theorem 2}

Suppose that $(f, g)$ is a pair of nonconstant meromorphic solution of (2). Then all zeros of $f$ and all 1-points of $f$ are poles of $g$, and poles of $f$ come from zeros, 1-points of $g$, or from zeros of $g^{\prime}$. By Nevanlinna's second fundamental theorem, we have

$$
\begin{aligned}
T(r, f) & \leq \bar{N}\left(r, \frac{1}{f}\right)+\bar{N}\left(r, \frac{1}{f-1}\right)+\bar{N}(r, f)+S(r, f) \\
& \leq \bar{N}(r, g)+\bar{N}\left(r, \frac{1}{g}\right)+\bar{N}\left(r, \frac{1}{g-1}\right)+\bar{N}\left(r, \frac{1}{g^{\prime}}\right)+S(r, f) \\
& \leq 5 T(r, g)+S(r, f) .
\end{aligned}
$$

Similarly, we have $T(r, g) \leq 5 T(r, f)+S(r, g)$. Therefore, $S(r, f)=S(r, g):=$ $S(r)$.

Suppose that $z_{0}$ is a zero of $f$ with multiplicity $n$. Then from (2), $z_{0}$ is a pole of $g$ with multiplicity $p$. Since the right-hand side of (2) is a nonzero constant, we have $2(k+1) n-1=(3 k+2) p+1$. If $p=1$, then $2(k+1) n=3(k+1)+1$, and thus 1 is divisible by $k+1$. This is impossible. Therefore, $p \geq 2$, and $2(k+1) n \geq$ $2(3 k+2)+2=6(k+1)$. Hence $n \geq 3$. This means that all zeros of $f$ have multiplicities $\geq 3$. Similarly, all zeros of $g$ have multiplicities $\geq 3$. By a similar argument, we can see that all 1-points of $f$ and $g$ have multiplicities $\geq 6$. And all poles of $f$ have multiplicities $\geq 2$ if they are not zeros of $g^{\prime}$. All poles of $g$ have multiplicities $\geq 2$ if they are not zeros of $f^{\prime}$. Denote by $N^{\prime}(r, f)$ the counting function of those poles of $f$ which are zeros or 1-points of $g$, and by $N^{\prime \prime}(r, f)$ the counting function of those poles of $f$ which are not zeros and 1-points of $g$. Then $N(r, f)=N^{\prime}(r, f)+N^{\prime \prime}(r, f)$ and $\bar{N}^{\prime \prime}(r, f) \leq \bar{N}\left(r, 1 / g^{\prime}\right)$. The notations $N^{\prime}(r, g)$ and $N^{\prime \prime}(r, g)$ are defined similarly. Using Nevanlinna's second fundamental theorem to the function $f$ and $g$, respectively, we get

$$
\begin{aligned}
& T(r, f) \leq \bar{N}^{\prime}(r, f)+\bar{N}^{\prime \prime}(r, f)+\bar{N}\left(r, \frac{1}{f}\right)+\bar{N}\left(r, \frac{1}{f-1}\right)-N_{0}\left(r, \frac{1}{f^{\prime}}\right)+S(r), \\
& T(r, g) \leq \bar{N}^{\prime}(r, g)+\bar{N}^{\prime \prime}(r, g)+\bar{N}\left(r, \frac{1}{g}\right)+\bar{N}\left(r, \frac{1}{g-1}\right)-N_{0}\left(r, \frac{1}{g^{\prime}}\right)+S(r),
\end{aligned}
$$


where $N_{0}\left(r, 1 / f^{\prime}\right)$ is the counting function of those zeros of $f^{\prime}$ which are not zeros and 1-points of $f$. The notation $N_{0}\left(r, 1 / g^{\prime}\right)$ is defined similarly. Note that $\bar{N}^{\prime \prime}(r, f) \leq N_{0}\left(r, 1 / g^{\prime}\right)$ and $\bar{N}^{\prime \prime}(r, g) \leq N_{0}\left(r, 1 / f^{\prime}\right)$. By adding the above two inequalities together, we get

$$
\begin{aligned}
T(r, f)+T(r, g) \leq & \bar{N}^{\prime}(r, f)+\bar{N}\left(r, \frac{1}{f}\right)+\bar{N}\left(r, \frac{1}{f-1}\right) \\
& +\bar{N}^{\prime}(r, g)+\bar{N}\left(r, \frac{1}{g}\right)+\bar{N}\left(r, \frac{1}{g-1}\right)+S(r) \\
\leq & \frac{1}{2} N^{\prime}(r, f)+\frac{1}{3} N\left(r, \frac{1}{f}\right)+\frac{1}{6} N\left(r, \frac{1}{f-1}\right) \\
& +\frac{1}{2} N^{\prime}(r, g)+\frac{1}{3} N\left(r, \frac{1}{g}\right)+\frac{1}{6} N\left(r, \frac{1}{g-1}\right)+S(r) \\
\leq & T(r, f)+T(r, g)+S(r) .
\end{aligned}
$$

The above inequalities show that "almost all" poles of $f$ and $g$ have multiplicities 2, "almost all" zeros of $f$ and $g$ have multiplicities 3, and "almost all" 1-points of $f$ and $g$ have multiplicities 6. Let

$$
\alpha_{1}=\frac{\left(f^{\prime}\right)^{6}}{f^{4}(f-1)^{5}}, \quad \alpha_{2}=\frac{\left(g^{\prime}\right)^{6}}{g^{4}(g-1)^{5}} .
$$

Then $\alpha_{1}$ and $\alpha_{2}$ are small functions of $f$ and $g$, and $\alpha_{1} \alpha_{1} \not \equiv 0$. The above equations imply $m(r, f)=S(r)$ and $m(r, g)=S(r)$. Let

$$
\alpha=f^{2}(f-1) g^{2}(g-1) .
$$

Then $\alpha$ is also a small function of $f$ and $g$, not identically zero. By considering the Laurent expansion of $f$ at a zero of $f$ with multiplicity 3, we can show that $\frac{\alpha_{1}}{\alpha_{2}}=\left(\frac{3}{2}\right)^{6} \alpha$. Similarly, by considering the Laurent expansions of $g$ at a zero of $g$ with multiplicity 3 , we get $\frac{\alpha_{2}}{\alpha_{1}}=\left(\frac{3}{2}\right)^{6} \alpha$. Therefore, $\alpha_{1}=\alpha_{2}$ or $\alpha_{1}=-\alpha_{2}$, and

$$
\alpha=\left(\frac{2}{3}\right)^{6} \frac{\alpha_{1}}{\alpha_{2}} \text {. }
$$

On the other hand, by considering the Laurent expansion of $f$ at a 1-point of $f$ with multiplicity 6 , we can obtain $\alpha=(1 / 3)^{6} \frac{\alpha_{1}}{\alpha_{2}}$, which contradicts the above equation. Hence equation (2) has no nonconstant meromorphic solutions.

\section{Proof of Theorem 3}

Suppose that $(f, g)$ is a pair of nonconstant meromorphic solution of (3). By an argument similar to that in the proof of Theorem 2, we can prove that "almost all" 
1-points and -1 points of $f$ and $g$ have multiplicities 4 , and "almost all" poles of $f$ and $g$ have multiplicities 2 . Let

$$
\beta_{1}=\frac{\left(f^{\prime}\right)^{4}}{(f-1)^{3}(f+1)^{3}}, \quad \beta_{2}=\frac{\left(g^{\prime}\right)^{4}}{(g-1)^{3}(g+1)^{3}} .
$$

Then $\beta_{1} \beta_{2} \not \equiv 0$. Both $\beta_{1}$ and $\beta_{2}$ are small functions of $f$ and $g$. Let

$$
\beta=(f-1)(f+1)(g-1)(g+1) .
$$

Then $\beta$ is also a small function of $f$ and $g$, and $\beta \not \equiv 0$. By considering the Laurent expansions of $f$ at a 1-point, and a -1 -point of $f$, respectively. We can show that $\beta_{1}=\beta_{2}=c^{2}$, and $\beta=1$. Then $f$ is a solution of the following equation:

$$
\left(f^{\prime}\right)^{4}=c^{2}(f-1)^{3}(f+1)^{3},
$$

and

$$
g=-\frac{1}{c} \frac{f\left(f^{\prime}\right)^{2}}{(f-1)^{2}(f+1)^{2}} .
$$

By Lemma 5, we get $f=1+\frac{(\wp-c / 2)^{2}}{c \wp}$, where $\wp$ is the $\wp$-function satisfies (6). Hence the conclusion can be proved easily.

\section{Proof of Corollary 1}

Let $F=H(f)=(f-a)^{n}(f-b)^{m}$ and $G=H(g)=(g-a)^{n}(g-b)^{m}$. Then we get $F^{\prime}=(n+m)(f-c)(f-a)^{n-1}(f-b)^{m-1} f^{\prime}$ and $G^{\prime}=(n+m)(g-c)(g-$ $a)^{n-1}(g-b)^{m-1} g^{\prime}$, where $c=(m a+n b) /(m+n)$. We have

$$
\begin{aligned}
N_{2}\left(r, F^{\prime}\right) & =2 \bar{N}(r, f) \leq 2 T(r, f), N_{2}\left(r, G^{\prime}\right)=2 \bar{N}(r, g) \leq 2 T(r, g), \\
N_{2}\left(r, \frac{1}{F^{\prime}}\right) & \leq 2 \bar{N}\left(r, \frac{1}{f-a}\right)+2 \bar{N}\left(r, \frac{1}{f-b}\right)+N_{2}\left(r, \frac{1}{f-c}\right)+N_{0}\left(r, \frac{1}{f^{\prime}}\right) \\
\leq & 4 T(r, f)+N_{0}\left(r, \frac{1}{f^{\prime}}\right)+N_{2}\left(r, \frac{1}{f-c}\right)+O(1),
\end{aligned}
$$

and

$$
N_{2}\left(r, \frac{1}{G^{\prime}}\right) \leq 4 T(r, g)+N_{0}\left(r, \frac{1}{g^{\prime}}\right)+N_{2}\left(r, \frac{1}{g-c}\right)+O(1)
$$

where $N_{0}\left(r, 1 / f^{\prime}\right)$ is the counting function of those zeros of $f^{\prime}$ that are not zeros of $(f-a)(f-b)(f-c)$. Note that $F^{\prime}$ and $G^{\prime}$ share the value $1 \mathrm{CM}$. If $F^{\prime} \neq G^{\prime}$ and $F^{\prime} G^{\prime} \neq 1$, then by Lemma 3 and inequalities (76), (77) and (78), we have 


$$
\begin{aligned}
T\left(r, F^{\prime}\right) \leq & 6 T(r, f)+N_{0}\left(r, \frac{1}{f^{\prime}}\right)+N_{2}\left(r, \frac{1}{f-c}\right)+6 T(r, g) \\
& +N_{0}\left(r, \frac{1}{g^{\prime}}\right)+N_{2}\left(r, \frac{1}{g-c}\right)+S(r, f)+S(r, g) .
\end{aligned}
$$

Since

$$
m\left(r, \frac{1}{F}\right) \leq m\left(r, \frac{1}{F^{\prime}}\right)+m\left(r, \frac{F^{\prime}}{F}\right)+O(1)
$$

we have

$$
T(r, F)-N\left(r, \frac{1}{F}\right) \leq T\left(r, F^{\prime}\right)-N\left(r, \frac{1}{F^{\prime}}\right)+S(r, f),
$$

which implies

$$
\begin{aligned}
(n+m) T(r, f) \leq & T\left(r, F^{\prime}\right)+\bar{N}\left(r, \frac{1}{f-a}\right)+\bar{N}\left(r, \frac{1}{f-b}\right) \\
& -N\left(r, \frac{1}{f-c}\right)-N_{0}\left(r, \frac{1}{f^{\prime}}\right)+S(r, f) .
\end{aligned}
$$

From this and (79), we get

$$
(n+m-8) T(r, f) \leq 9 T(r, g)+S(r, f)+S(r, g) .
$$

Symmetrically, we have

$$
(n+m-8) T(r, g) \leq 9 T(r, f)+S(r, f)+S(r, g) .
$$

These two inequalities yield

$$
(n+m-17)(T(r, f)+T(r, g)) \leq S(r, f)+S(r, g),
$$

which is impossible when $n+m \geq 18$. Hence $F^{\prime} G^{\prime}=1$ or $F^{\prime}=G^{\prime}$.

By Theorem 1, we can rule out the case $F^{\prime} G^{\prime}=1$. Therefore, we have $F^{\prime}=G^{\prime}$, and thus there exists a constant $\mathrm{C}$ such that $H(f)=H(g)+C$, i.e., $(f-a)^{n}(f-$ $b)^{m}=(g-a)^{n}(g-b)^{m}+C$. This implies $T(r, f)=T(r, g)+O(1)$. If $C \neq 0$, then by the second fundamental theorem, we get

$$
T(r, H(f)) \leq \bar{N}(r, H(f))+\bar{N}\left(r, \frac{1}{H(f)}\right)+\bar{N}\left(r, \frac{1}{H(f)-C}\right)+S(r, H(f)),
$$

which yields

$$
\begin{aligned}
(n+m) T(r, f) \leq & \bar{N}(r, f)+\bar{N}\left(r, \frac{1}{f-a}\right)+\bar{N}\left(r, \frac{1}{f-b}\right) \\
& +\bar{N}\left(r, \frac{1}{g-a}\right)+\bar{N}\left(r, \frac{1}{g-b}\right)+S(r, f) .
\end{aligned}
$$


From the above inequality, we get $n+m \leq 5$, which contradicts the assumption. Therefore, $C=0$. It follows that $(f-a)^{n}(f-b)^{m}=(g-a)^{n}(g-b)^{m}$, i.e.,

$$
\left(\frac{f-a}{g-a}\right)^{n}=\left(\frac{g-b}{f-b}\right)^{n} \text {. }
$$

Since $m$ and $n$ are relatively prime, there exist two integers $u$ and $v$ such that $u m+v n=1$. Let $h=[(f-a) /(g-a)]^{u}[(g-b) /(f-b)]^{v}$. It follows from the above equation that $(f-a) /(g-a)=h^{m},(g-b) /(f-b)=h^{n}$. From these two equations, we see that $h$ is not constant and

$$
f=\frac{b h^{m+n}+(a-b) h^{m}-a}{h^{m+n}-1}, \quad g=\frac{a h^{m+n}+(b-a) h^{m}-b}{h^{m+n}-1},
$$

which completes the proof of Corollary 1.

\section{REFERENCES}

1. I. N. Baker, On a class of meromorphic functions, Proc. Amer. Math. Soc., 17 (1966), 819-822.

2. F. Gross, On the equation $f^{n}+g^{n}=1$, Bull. AMS., 72 (1966), 86-88.

3. G. G. Gundersen and K. Tohge, Entire and meromorphic solutions of $f^{5}+g^{5}+h^{5}=1$, Symposium on Comlex Differential and Functional Equations, Univ. Joensuu Dept. Math. Report. Ser. No. 6, 2004, pp. 57-67.

4. W. Hayman, Meromorphic Functions, Oxford, Clarendon Press, 1964.

5. H. K. Ha and C.-C. Yang, On the functional equation $P(F)=Q(G)$, Value Distribution Theory and Related Topics, Book series: Advanced in Complex Analysis and Its Applications 3, Kluwer Academic Publishers, 2004, pp. 201-208.

6. E. Mues and M. Reinders, Meromorphic functions sharing one value and unique range sets, Kodai Math. J., 18 (1995), 515-522.

7. P. C. Hu, P. Li and C.-C. Yang, Unicity of Meromorphic Mappings, Kluwer Academic Publishers, Dordrecht, 2003.

8. C.-C. Yang, A generalization of a theorem of P. Montel on entire functions, Proc. $A M S, \mathbf{2 6}(2)$ (1970), 332-334.

9. C.-C. Yang and X.-H. Hua, Uniqueness and value sharing of meromorphic functions, Ann. Acad. Sci. Fennicae Math., 22 (1997), 395-406.

10. C.-C. Yang and P. Li, Some further results on the functional equation $P(f)=Q(g)$, Value Distribution Theory and Related Topics, Book series: Advanced in Complex Analysis and Its Applications 3, Kluwer Academic Publishers, Dordrecht, 2004, pp. 219-231. 
11. Valiron, G., Sur la dérivée des fonctions algébroides, Bull. Soc. Math. France, 59 (1931), 17-39.

Mingbo Yang and Ping Li

Department of Mathematics

University of Sciences and Technology of China

Hefei, Anhui 230026

P. R. China

E-mail: mbyang@mail.ustc.edu.cn pli@ustc.edu.cn 
